\title{
Eine neue Nitroprussidreaktion des Harnes.
}

\author{
Von \\ V. Arnold.
}

(Aus der Abteilung für Infektionskrankheiten des Allg. Krankenhauses in Lemberg.)

(Der Redaktion zugegangen am 2. Oktober 1906.)

Man beobachtet die in folgendem beschriebene sehr charakteristische Reaktion nach dem Genuß von Fleisch oder Fleischbrühe. Sie wird in folgender Weise ausgeführt: 10-20 ccm des betreffenden Harnes versetzt man in einem Kelchgläschen mit einem Tropfen einer 40\%igen Nitroprussidnatriumlösung und darauf mit 5-10 ccm einer 5\% igen Natron- oder Kalilauge. Es tritt zuerst ein kräftiges und reines Violett auf, welches alsbald in purpurrot und sodann allmählich (im Verlauf einer Minute etwa) durch braunrot in gelb übergeht.

Will man diese Reaktion in ihrer größten Farbenreinheit beobachten, so ist der Harn vorher durch Tierkohle zu entfärben. Die Intensität der violetten Reaktion erfährt dadurch keine Einbuße; im Gegenteil fällt die Reaktion mit dem wasserklaren Filtrat - sogar bei geringerer Intensität derselben deutlicher und farbenreiner aus, da der störende Einfluß des gelben Harnpigmentes wegfällt. Übrigens fällt diese Reaktion auch mit nativem Harn durchaus intensiv und deutlich aus. Die Entfärbung des Harnes darf deshalb für gewöhnlich als überflüssig entfallen; nur zum Studium der Reaktion an und für sich ist sie zu empfehlen.

Die violette resp. purpurviolette Flüssigkeit zeigt ein $\mathrm{Ab}$ sorptionsband zwischen D und E, welches bei stärkerer Konzentration sogar über $\mathrm{b}$ hinausreicht. Das Maximum der $\mathrm{Ab}-$ sorption beginnt gleich hinter D und erreicht nicht $\mathrm{E}$.

Die violette resp. purpurrote Farbe dieser Reaktion geht auf Zusatz von Essigsäure in blau über, welches noch rascher als das Violett der alkalischen Lösung verblaßt und schließlich in einen blaßgelblichen Farbenton übergeht. Diese Flüchtigkeit 
der Reaktion erschwert auch die spektroskopische Untersuchung derselben, doch kann man beobachten, daß die tiefblaue Lösung auch ein - im Vergleich zur alkalischen violetten schwächeres - Absorptionsband besitzt, welches auf D liegt (ein wenig vor $D$ beginnend) und sich etwas über D nach rechts hin erstreckt. Das spektrale Rot ist leicht absorbiert. Die Flüchtigkeit dieser Reaktion kann übrigens durch vorherige Kühlung des Harnes und der Natronlauge vermindert werden. Die Reaktion dauert dann 3-4 Minuten, während sie sonst in 1 Minute (bei höherer Außentemperatur sogar in einer $1 / 2$ Minute) verblaßt ist.

Man kann die Natronlauge bei Ausführung dieser Reaktion auch durch Ammoniak ersetzen, jedoch genügt in diesem Fall nicht ein Tropfen der 4-5\% igen Nitroprussidnatriumlösung, sondern eine deutliche Reaktion erhält man erst nach Zufügung von 5-6 Tropfen dieser Lösung auf 5-6 ccm Harn; ferner muß der Harn vorher durch Tierkohle filtriert werden, da die violette Reaktion mit $\mathrm{NH}_{3}$ überhaupt bedeutend schwächer ausfällt, weshalb bei Verwendung von nativem Harn das Violett unrein und matt erscheint. Das bei dieser Reaktion zuerst auftretende Violett verblaßt nach kurzer Zeit, indem es zugleich durch das später auftretende und allmählich sich verstärkende Rot der Weylschen Kreatininreaktion ersetzt wird. Wurde die Reaktion an einem Harn angestellt, der nur Kreatinin enthielt, so erhält man nur diesen zweiten Teil der Reaktion. In gleicher Weise verhalten sich reine Kreatininlösungen. Wir sehen hier übrigens, daß die violette Reaktion zeitlich der Kreatininreaktion vorangeht.

(Die Angabe Weyls, daß die nach ihm benannte Kreatininreaktion mit $\mathrm{NH}_{3}$ nicht eintrete, ist unrichtig. Die Reaktion fällt zwar schwächer aus, sie persistiert dafür aber durch längere Zeit. Auch ist das Rot der Weylschen Reaktion bei Anwendung von $\mathrm{NH}_{3}$ reiner als bei Anwendung von Natronlauge.)

Bei geringerer Intensität der Reaktion kann das Violett derselben durch Purpurviolett oder Purpurrot ersetzt sein. Will man das Violett in solchen Fällen dennoch zur Anschauung bringen, so wird man den Harn entfärben. Die W eylsche Kreatininreaktion ist übrigens auch in diesem Falle 
nicht mit der nach Fleischgenuß auftretenden Reaktion zu verwechseln.

Diese so charakteristische Reaktion wurde bisher trotz der Häufigkeit ihres Auftretens im Harn übersehen. Es geschah dies deshalb, weil das gleichzeitige Auftreten einer intensiven Kreatininreaktion die violette Reaktion, welche nur um einen Moment der Weylschen Reaktion vorangeht, verdeckt.

Erst die Beobachtung, daß das Optimum beider Reaktionen einem bestimmten, durchaus verschiedenen Zusatz von Nitroprussidnatrium entspricht, ermöglichte eine Trennung beider Reaktionen und eine gesonderte Beobachtung derselben.

Die von mir beschriebene violette Reaktion erfordert eine relativ geringe Quantität der 40\% igen Nitroprussidnatriumlösung, denn sie tritt bereits in voller Intensität auf Zusatz eines Tropfens dieser Lösung auf 15-20 ccm Harn auf. Unter diesen Umständen reagiert aber das Kreatinin des Harnes bei gleichzeitiger Anwesenheit des die violette Reaktion gebenden Körpers, wenn überhaupt, so in kaum sichtbarer Weise, da die violette Reaktion der Weylschen Kreatininreaktion zeitlich vorangeht; in keinem Fall wird aber durch dasselbe der Verlauf jener Reaktion störend beeinflußt.

Wird mehr Nitroprussidnatrium zugesetzt, so bemerkt man auch, daß der störende Einfluß der fast gleichzeitig auftretenden Weylschen Reaktion immer mehr zur Geltung gelangt.

Versetzt man nun $10-15 \mathrm{ccm}$ einer $0,1-0,5 \%$ igen Kreatininlösung mit einem Tropfen einer 4\% igen Nitroprussidnatriumlösung, so erhält man auf Zusatz von 5-10 ccm einer $5 \%$ igen Natronlauge nur eine schwache Rotfärbung. (So färbt sich eine 0,1\% oige wässerige Kreatininlösung mit dem Reagens schwach gelbrötlich, nur durch die rötliche Nuance unterscheidbar von dem Verhalten einer Lösung von 1 Tropfen einer 4\% \% igen Nitroprussidnatriumlösung auf $15 \mathrm{ccm}$ Wasser; fast ebenso verhält sich eine 0,2\% ige Lösung. Erst eine 0,4\% ige Lösung gibt unter diesen Umständen eine deutlichere, wenn auch immerhin nur schwache Rotfärbung, die alsbald in gelb- 
rot und schließlich in gelb übergeht. Diese Konzentration der Kreatininlösung dürfte dem durchschnittlichen, prozentischen Gehalt des normalen und pathologischen Harnes an Kreatinin entsprechen.) Eine intensive Kreatininreaktion erzielt man erst bei Anwendung von etwa einem Tropfen einer 10\% igen Nitroprussidnatriumlösung auf $1 \mathrm{ccm}$ Harn oder Kreatininlösung unter Zusatz einer 10\% igen Natronlauge. (Es entspricht dies also einer etwa 20 mal so großen Quantität von Nitroprussidnatrium, als zur Erlangung der maximalen Intensität der von mir beschriebenen Reaktion notwendig erscheint.) Die Reaktion verläuft dann in der Weise, daß die Flüssigkeit zuerst vorübergehend rot, dann rotgelb und schließlich gelb wird. Untersucht man die Farbenreaktion des Kreatinins mit dem Spektroskop, so vermißt man jedes Absorptionsband. Die rote Lösung absorbiert das Spektrum von violett bis nahe an D. Wird die Probe mit Essigsäure übersättigt, so entfärbt sie sich sogleich und die Färbung der Mischung wird grünlich. Diese Kreatininreaktion ist so gänzlich verschieden von der von mir beschriebenen, violetten Reaktion, daß ein Zweifel daran nicht einmal aufkommen kann. Harne, welche nur Kreatinin, wenn auch in vermehrter Quantität enthalten, verhalten sich wie eine $0,1-0,5 \%$ ige Kreatininlösung, d. i. sie werden mit einem Tropfen einer 4\% $\%$ igen Nitroprussidnatriumlösung unter Zusatz 5\% iger Natronlauge nur eine schwache (bei stärkerer Eigenfarbe des Harnes oft kaum sichtbare) Rotfärbung zeigen, während auf Zusatz einer entsprechenden Quantität der Nitroprussidnatriumlösung in demselben Harn oft eine intensive Kreatininreaktion zur Beobachtung gelangt. So verhält sich gewöhnlich der Harn Typhöser im Stadium der Kontinua, wenn die Kranken durch einige Zeit Fleischbrühe nicht erhielten oder zurückgewiesen hatten. Es wurden gegen 40-50 solcher Harne untersucht, in welchen keine Spur des die violette Reaktion gebenden Körpers gefunden werden konnte. Es wäre daher eine ,endogene“ Entstehung dieses Körpers im Stoffwechsel des Organismus ausgeschlossen.

Alkalisiert man eine Harnprobe mit Kali- oder Natronlauge, so erhält man bereits nach sehr kurzer Zeit (d. i. nach 
etwa 20-30 Sekunden) nur noch die gewöhnliche Kreatininreaktion, da die die violette Reaktion hervorrufende Substanz durch das Alkali zersetzt wird, während das Kreatinin in dieser kurzen Zeit durch das Alkali natürlich nicht beeinflußt wird. Schwächer und erst nach längerer Zeit wirkt $\mathrm{NH}_{3}$ und $\mathrm{Ba}(\mathrm{OH})_{2}$. Man könnte diesen Umstand dazu benützen, um eine schwache Reaktion von der Weylschen Reaktion zu unterscheiden, da man letztere nach Eliminierung der die violette Reaktion gebenden Substanz für sich gesondert vornehmen kann.

Diese Reaktion wird ferner durch Aufkochen nach Zusatz einer Mineralsäure aufgehoben. während ein auch durch einige Minuten fortgesetztes Kochen der Probe die Intensität der Reaktion nicht vermindert. Eine Verminderung der Intensität derselben wurde auch nach Ausfällung des Harnes vermittelst basischen Bleiacetats nicht beobachtet. In das Destillat geht diese mit Nitroprussidnatrium reagierende Substanz nicht über. Es gelang nicht, dem nativen oder dem mit Säure versetzten Harne durch die verschiedensten Lösungsmittel (Äthyläther, Essigäther, Amylalkohol, Benzol, Chloroform) diese Substanz zu entziehen. Nur von Alkohol wird sie aufgenommen. Der durch Tierkohle entfärbte Harn wird zu diesem Zwecke unter Erwärmen mit Ammonsulfat gesättigt, und darauf mit Alkohol geschüttelt. Die Reaktion tritt in diesem fast farblosen oder schwach gelblichen alkoholischen Extrakt sehr intensiv und rein auf. Nach Zusatz von Nitroprussidnatrium und Natronlauge beobachtet man ein schönes Blauviolett, welches in purpurrot und schließlich in gelb übergeht. Nachdem die Reaktion verblaßt ist, kann man durch weiteren Zusatz von Nitroprussidnatrium durch das Auftreten einer meist ziemlich intensiven W eylschen Reaktion auch die Anwesenheit von Kreatinin beweisen. Der mit konzentrierter Ammonsulfatlösung versetzte Harn reagiert mit einem Tropfen einer $4 \%$ oigen Nitroprussidnatriumlösung auf $15 \mathrm{ccm}$ Harn nicht mehr; erst nach Zusatz einiger Tropfen dieser Lösung erfolgt eine sehr schwache Reaktion, da die Gegenwart von Ammonsalzen sowohl die Weylsche Kreatininreaktion, als auch die nach Fleischgenuß auftretende violette Reaktion stark beeinträchtigt, während der 
Zusatz einer konzentrierten Kochsalzlösung oder einer Lösung von essigsaurem Natrium diese Reaktionen nicht verhindert. Wird der Harn durch einige Zeit aufbewahrt, so büßt die Reaktion nach und nach an Intensität ein, ist aber auch nach einigen Wochen noch nachweisbar. Nach längerer Zeit erst verschwindet die Reaktion und zwar früher, als die Weylsche Kreatininreaktion.

Den bedeutendsten Gehalt an dieser mit Nitroprussidnatrium reagierenden Substanz enthält der Harn - wie ich schon eingangs erwähnte - nach Genuß einer kräftigen Fleischbrühe (besonders von sog. Beeftea, welches aus $1 \mathrm{~kg}$ Fleisch bereitet wurde), sowie von gebratenem Fleisch. Man kann jedoch nachweisen, daß die fragliche Substanz im Beeftea nicht präformiert ist, da man die charakteristische violette Reaktion mit demselben nicht erhält. Nach Genuß von rohem oder nur kurze Zeit einer höheren Temperatur ausgesetztem Fleisch (Backhuhn etc.) erreicht die Intensität der Reaktion nicht annähernd den Grad, wie nach Genuß von gebratenem oder geschmortem Fleisch. Auch nach Genuß von gebratener Leber war dieser Körper an der intensiven violetten Reaktion mit Nitroprussidnatrium im Harn nachweisbar, während nach Genuß von Gehirn - wie vorauszusehen war - nur eine schwache Reaktion erhalten wurde.

In Betreff der zeitlichen Verhältnisse der Ausscheidung dieser Substanz konnte ich feststellen, daß die Hauptmenge derselben in dem ersten 2-3 Stunden nach Genuß von Fleischbrühe und Fleisch entleerten Harn enthalten ist. Doch wird dieselbe auch noch in den folgenden Stunden im Harn ausgeschieden; 24 Stunden nach dem Genuß eines Fleischgerichtes war nur noch eine sehr schwache Reaktion zu konstatieren.

Die Ausscheidung dieser Substanz folgt übrigens sehr rasch auf die Aufnahme von Fleischbrühe; während 10 Minuten nach Genuß derselben nur erst eine sehr schwache Reaktion zu erhalten ist, konnte bereits 10 Minuten später (d. i. 20 Minuten nach Aufnahme der Fleischbrühe) eine intensive Reaktion erhalten werden. Eine $1 / 2$ bis $3 / 4$ Stunde nach Genuß der Fleischbrühe hatte die Ausscheidung bereits ihr Maximum er- 
reicht; sie dauert aber auch viel kürzer, als nach Aufnahme eines Fleischgerichtes.

Die Reaktion ist übrigens in jedem Harn nach dem Mittagessen (wenn dasselbe nur aus Fleischbrühe und einem Stück Fleisch besteht) mit großer Deutlichkeit nachweisbar und muß dieser Umstand bei Anstellung der W eylschen Kreatininreaktion berücksichtigt werden. Wird weder Fleisch noch Fleischbrühe genossen, so verschwindet die Substanz vollkommen.

Während man also beim Kreatinin eine exogene und endogene Entstehung unterscheiden muß, ist nach solchen Erfahrungen bei unserer Substanz ein endogener Ursprung derselben ausgeschlossen.

Außer dem Kreatinin reagiert unter den Harnbestandteilen mit Nitroprussidnatrium und Alkali noch die Acetessigsäure und das Aceton. Von diesen beiden Körpern interessiert uns in harnanalytischer Hinsicht vorzüglich die Acetessigsäure, da die Acetonreaktionen mit Nitroprussidnatrium in frischentleertem Harn nach meinen Erfahrungen (Zentralbl. f. inn. Med. 1900) durch die Acetessigsäure bedingt werden. Übrigens verhalten sich gegen Nitroprussidnatrium beide Körper in identischer Weise.

Bei Verwendung einiger Tropfen einer 10\% igen Nitroprussidnatriumlösung auf ebensoviel Kubikzentimeter einer etwa $5 \%$ igen Acetonlösung erhält man auf Zusatz einer 10\%igen Natronlauge eine intensive Rotfärbung, die alsbald in orangerot übergeht und allmählich in gelb verblaßt. Übersättigt man die Reaktionsmischung, so lange die rote Farbe noch nicht in gelb verblaßt ist, mit Essigsäure, so wandelt sich die Farbe in ein relativ beständiges Purpurrot (Legals Reaktion). Von einer Ähnlichkeit dieser Reaktion mit der von mir beschriebenen kann daher - wie ersichtlich - nicht die Rede sein.

Die von mir beobachtete violette Reaktion wird übrigens durch die gleichzeitige Anwesenheit von Acetessigsäure oder Aceton im Harn nicht beeinträchtigt, da diese Verbindungen mit 1 Tropfen einer 40\%igen Nitroprussidnatriumlösung $\mathrm{zu}$ schwach reagieren, um eine Störung derselben verursachen zu können. Endlich ergeben die Ammonsalze (Chlorammonium, 
schwefelsaures Ammon, kohlensaures Ammon, essigsaures, milchsaures Ammon, Rhodanammonium u. a.) mit Nitroprussidnatrium und Ammoniak (nicht aber mit Nitroprussidnatrium und fixem Alkali) ebenfalls eine violette Farbenreaktion, welche auch ein Absorptionsband zwischen $\mathrm{D}$ und $\mathrm{b}$ zeigt. Diese Reaktion erhält man am intensivsten mit Ammoniak vom spez. G. 0,875 bei Anwendung konzentrierter Lösungen eines Ammonsalzes (weniger intensiv erzielt man sie mit $\mathrm{NH}_{3}$ vom sp. $\mathrm{G}$. 0,910). Eine Lösung von $1 \mathrm{ccm}$ kaltgesättigter Chlorammonlösung auf $10 \mathrm{ccm}$ aqua destillata mit $\mathrm{NH}_{3}$ von 0,910 spez. G. ergibt nur eine schwache Reaktion, während nach Verdünnung dieses Ammoniaks mit dem gleichen Volumen Wasser eine Reaktion nicht mehr auftritt. Diese Reaktion erreicht erst nach einigen Minuten ihr Intensitätsmaximum und persistiert sodann an lichtgeschützter Stelle durch längere Zeit, während welcher das Violett allmählich in blauviolett und schließlich in schwarzblau übergeht. In direktem Sonnenlicht verblaßt die Farbe rasch. Auch diese Reaktion tritt bei Anwendung von 1 Tropfen einer $4-5 \%$ igen Nitroprussidnatriumlösung auf $5-10 \mathrm{ccm}$ einer konzentrieten Ammonsalzlösung nur schwach auf. Eine intensive Reaktion wird erst bei Anwendung von etwa 1 Tropfen einer 10\% igen Nitroprussidnatriumlösung auf $1 \mathrm{ccm}$ Lösung

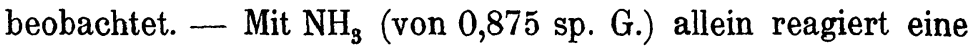
Nitroprussidnatriumlösung nicht (die Lösung verändert nur ihre Farbe nach einigen Minuten in gelblich-braun). Wird aber durch Zusatz einiger Tropfen einer Säure (z. B. konzentrierter $\mathrm{HCl})$ die Bildung eines Ammonsalzes veranlaßt, so tritt die Reaktion sogleich in typischer Weise auf. - Im unzersetzten Harn gelangt diese Reaktion nicht zur Beobachtung.

Von sonstigen mit Nitroprussidnatrium reagierenden Verbindungen wäre hier noch das Cystein zu erwähnen, welches nach K. Mörner auf Zusatz von Nitroprussidnatrium und Alkali mit purpurroter Farbe, die durch rot in gelb übergeht, reagiert. Wird Essigsäure im ersten Stadium zugesetzt, so tritt Entfärbung ein. Auch der Farbenton dieser Reaktion ist von der violetten Farbe der von mir beschriebenen Reaktion vollkommen verschieden. 
Das Indol, welches eine ähnliche Reaktion mit Nitroprussidnatrium ergibt, kommt als solches im Harn nicht vor. Die Nitroprussidreaktion des Indols verläuft in folgender Weise: (zur Verwendung gelangte eine alkoholische 0,1\% ige Lösung von E. Merck). Diese mit einigen Tropfen einer $40 \%$ igen Nitroprussidnatriumlösung versetzte Lösung reagiert auf Zusatz einer 5-10\% igen Natronlauge mit purpurvioletter Farbe. Diese Reaktion besitzt durchaus nicht die charakteristische Flüchtigkeit der von mir beschriebenen Reaktion, da sie unverändert durch lange Zeit persistiert und erst nach längerer Zeit durch purpurrot in braunrot und braun übergeht. Wird Essigsäure zugesetzt, so wandelt sich die purpurviolette Färbung in reinblau um. Auch dieses Blau zeichnet sich im Gegensatz zu der Flüchtigkeit der entsprechenden Reaktionsphase der von mir beschriebenen Reaktion durch große Beständigkeit aus, da es tagelang unverändert persistiert. Abweichend ist ebenfalls das spektroskopische Verhalten dieser Reaktion.

$\mathrm{Zu}$ erwähnen wäre noch das Methylmerkaptan, welches jedoch mit Nitroprussidnatrium in anderer Weise reagiert, als der uns hier interessierende Körper, welcher außerdem in das Destillat nicht übergeht.

Die von mir beschriebene Nitroprussidreaktion des Harnes ist nach dem Vorgebrachten verschieden von den bisher beschriebenen Nitroprussidreaktionen des Harnes, welchen sie an praktischer Bedeutung gewiß nicht nachsteht, da sie uns in den Stand setzt, objektiv den stattgefundenen Genuß von Fleisch oder Fleischbrühe nachzuweisen, und auf diese Weise eine Überschreitung unserer Diätvorschriften zu erkennen.

Lemberg, den 28. IX. 1906. 